\title{
Some properties of algebraic difference equations of first order
}

\section{Yong Liu* (1)}

\section{"Correspondence: \\ liuyongsdu1982@163.com \\ Department of Mathematics, \\ Shaoxing College of Arts and \\ Sciences, Shaoxing, Zhejiang \\ 312000, P.R. China}

\section{Abstract}

We prove that if $g(z)$ is a finite-order transcendental meromorphic solution of

$$
\left(\triangle_{c} g(z)\right)^{2}=A(z) g(z) g(z+c)+B(z),
$$

where $A(z)$ and $B(z)$ are polynomials such that $\operatorname{deg} A(z)>0$, then

$$
1 \leq \rho(g)=\max \left\{\lambda(g), \lambda\left(\frac{1}{g}\right)\right\} .
$$

MSC: $30 \mathrm{D} 35 ; 39 \mathrm{~B} 12$

Keywords: meromorphic functions; difference equations; value distribution; finite order

\section{Introduction}

Steinmetz [1] and Bank and Kaufman [2] proved that the equation

$$
\left(g^{\prime}\right)^{n}=R(z, g)
$$

can be reduced into a list of six simple differential equations by a suitable Möbius transformation with polynomial coefficients, which include

$$
\left(g^{\prime}\right)^{2}=p(z)(g-q(z))^{2}(g-\zeta)(g-\eta)
$$

where $\zeta, \eta$ are constant, and $p(z), q(z)$ are rational functions. Let $q(z) \in \mathbb{C}$. Then equation (1.1) can be transformed into

$$
\left(g^{\prime}\right)^{2}=P(z)\left(g^{2}-1\right)
$$

Ishizaki and Korhonen [3] investigated meromorphic solutions of

$$
(\triangle g(z))^{2}=P(z)(g(z) g(z+1)-Q(z)) .
$$
(http://creativecommons.org/licenses/by/4.0/), which permits unrestricted use, distribution, and reproduction in any medium, provided you give appropriate credit to the original author(s) and the source, provide a link to the Creative Commons license, and indicate if changes were made. 
They proved that equation (1.2) possesses a continuous limit to the equation

$$
\left(g^{\prime}\right)^{2}=P(z)\left(g^{2}-1\right)
$$

which extends to solutions in certain cases.

We assume that the reader is familiar with the basic notions of Nevanlinna theory (see, e.g., $[4,5])$. Of late, several scholars [3, 6-14] studied the properties of finite-order meromorphic solutions of algebraic difference equations and obtained many interesting results.

For the special case of (1.2), Whittaker [15] has shown that the equation

$$
g(z+1)=q(z) g(z)
$$

where $q(z)$ is a meromorphic function of finite order $\rho(q)$, has a meromorphic solution $g$ such that $\rho(q) \leq \rho(g) \leq \rho(q)+1$. Here $\rho(g)$ denotes the order of growth of the meromorphic function $g(z)$.

Chen [7] has extended this above result and proved that the Pielou logistic equation

$$
g(z+1)=\frac{R(z) g(z)}{P(z)+Q(z) g(z)},
$$

where $R(z), P(z)$, and $Q(z)$ are polynomials with $P(z) R(z) Q(z) \not \equiv 0$, has a finite-order transcendental meromorphic solution $g$ such that $1 \leq \rho(g)$.

Replacing $g(z+1)$ with $\triangle g(z)$, Ishizaki [10] was concerned with the growth and value distributions of transcendental meromorphic solutions of the algebraic difference equation

$$
(\triangle g(z))^{2}=P(z) g(z)
$$

In 2014, Liu [12] considered the Nevanlinna growth of an equation related to (1.2). It is interesting to consider some properties of (1.2), and our results will be stated in Section 2 .

\section{Main results}

Theorem 2.1 Let $c \in \mathbb{C} \backslash\{0\}$, and let $A(z)$ and $B(z)$ be polynomials such that $\operatorname{deg} A(z)>0$. If $g(z)$ is a finite-order transcendental meromorphic solution of

$$
\left(\triangle_{c} g(z)\right)^{2}=A(z) g(z) g(z+c)+B(z)
$$

then

$$
1 \leq \rho(g)=\max \left\{\lambda(g), \lambda\left(\frac{1}{g}\right)\right\} .
$$

Remark It is a curious problem to construct a transcendental meromorphic solution of (2.1) for the case $\operatorname{deg} A>0$.

Theorem 2.2 Let $c \in \mathbb{C} \backslash\{0\}$, and let $E(z)=\frac{D(z)}{F(z)}$ be an irreducible rational function, where $D(z)$ and $F(z)$ are polynomials with $\operatorname{deg} D(z)=d$ and $\operatorname{deg} F(z)=f$. If the equation

$$
\left(\triangle_{c} g(z)\right)^{2}=g(z) g(z+c)+E(z)
$$


has a rational solution

$$
g(z)=\frac{H(z)}{K(z)}=\frac{l_{h} z^{h}+\cdots+l_{0}}{m_{k} z^{k}+\cdots+m_{0}},
$$

where $l_{h}(\neq 0), \ldots, l_{0}, m_{k}(\neq 0), \ldots, m_{0}$ are constants, $\operatorname{deg} H(z)=h$, and $\operatorname{deg} K(z)=k$.

(i) If $d \geq f$ and $d-f$ is zero or an even number, then

$$
h-k=\frac{d-f}{2} .
$$

(ii) If $d<f$, then $h-k=\frac{d-f}{2}$.

Further, Example 2.3 shows that there exist rational solutions satisfying Theorem 2.2(i), and Example 2.4 shows that there exist rational solutions satisfying Theorem 2.2(ii).

Example 2.3 The equation

$$
(g(z+c)-g(z))^{2}=g(z+c) g(z)+c^{2}-z^{2}-(4+c) z-2 c-4
$$

has a rational solution $g(z)=z+2$, where $d=2, f=0$, and $h-k=1=\frac{d-f}{2}$.

Example 2.4 The equation

$$
(g(z+c)-g(z))^{2}=g(z+c) g(z)+\frac{c^{2}-z(z+c)}{z^{2}(z+c)^{2}}
$$

has a rational solution $g(z)=\frac{1}{z}$, where $d=2, f=4$, and $h-k=-1=\frac{d-f}{2}$.

\section{Proof of Theorem 2.1}

Lemma 3.1 ([11]) Let $w(z)$ be a transcendental meromorphic solution of finite order of the difference equation

$$
P(z, w)=0,
$$

where $P(z, w)$ is a difference polynomial in $w(z)$ and its shift. If $P(z, a) \not \equiv 0$ for a slowly moving target function $a$, that is, $T(r, a)=S(r, w)$, then

$$
m\left(r, \frac{1}{w-a}\right)=S(r, w) .
$$

The following result obtained by Chiang and Feng [16] and Halburd and Korhonen [9, 17] independently. We state here the form stated in [16, Theorem 8.2(b)].

Lemma 3.2 ([16]) Let $c_{1}, c_{2}$ be two arbitrary complex numbers, and let $w(z)$ be a meromorphic function of finite order $\rho$. Let $\varepsilon>0$ be given. Then there exists a subset $E \subset(1, \infty)$ of finite logarithmic measure such that, for all $|z|=r \notin E \cup[0,1]$, we have

$$
\exp \left(-r^{(\rho-1+\varepsilon)}\right) \leq\left|\frac{w\left(z+c_{1}\right)}{w\left(z+c_{2}\right)}\right| \leq \exp \left(r^{(\rho-1+\varepsilon)}\right)
$$


Firstly, we prove that $\rho(g)=\rho \geq 1$. We consider the following two cases separately.

Case 1.1. If $g(z)$ has infinitely many poles, we can pick a pole $z_{0}$ of $g(z)$ such that $g\left(z_{0}\right)=$ $\infty^{\pi}$, where $\pi \geq 1$, then we deduce by (2.1) that $g\left(z_{0}+c\right)=\infty^{\pi_{1}}$, where $\pi_{1} \geq m$. Substituting $z_{0}+c$ for $z$ into (2.1), we have

$$
(g(z+2 c)-g(z+c))^{2}=A(z+c) g(z+2 c) g(z+c)+B(z+c) .
$$

Then (3.1) implies that $z_{0}+2 c$ is a pole of $g$ of multiplicity $\pi_{2} \geq \pi_{1} \geq \pi$.

Since $g(z)$ has infinitely many poles, following the previous steps, we pick a pole $z_{0}$ of $g(z)$ such that

$$
g\left(z_{0}+n c\right)=f\left(\xi_{n}\right)=\infty^{\pi_{n}}
$$

where $\pi_{n} \geq \pi$ for all $n \in \mathbb{N}^{0}$. Hence, we can choose a sequence $\left\{\xi_{n}=z_{0}+n c, n \in \mathbb{N}^{0}\right\}$ of poles of $g(z)$, the multiplicity of which is $\pi_{n} \geq \pi$, so we obtain $\lambda\left(\frac{1}{g}\right) \geq 1$, and therefore $\rho(g) \geq \lambda\left(\frac{1}{g}\right) \geq 1$.

Case 1.2. If $g(z)$ is a transcendental meromorphic function with finitely many poles, then we can rewrite $g(z)$ as

$$
g(z)=\frac{g_{1}(z)}{P(z)}
$$

where $g_{1}(z)$ is a transcendental entire function, and $P(z)$ is a polynomial. Substituting (3.2) into (2.1), we have

$$
\left(\frac{g_{1}(z+c)}{P(z+c)}-\frac{g_{1}(z)}{P(z)}\right)^{2}=A(z) \frac{g_{1}(z+c)}{P(z+c)} \frac{g_{1}(z)}{P(z)}+B(z) .
$$

By computing (3.3) we have

$$
\frac{P(z)}{P(z+c)} \frac{g_{1}(z+c)}{g_{1}(z)}+\frac{P(z+c)}{P(z)} \frac{g_{1}(z)}{g_{1}(z+c)}=2+A(z)+\frac{B(z) P(z) P(z+c)}{g_{1}(z) g_{1}(z+c)} .
$$

We prove that $\rho(g)=\rho\left(g_{1}\right)=\rho \geq 1$. Suppose, on the contrary to the assertion, that $\rho(g)=$ $\rho\left(g_{1}\right)=\rho<1$. For any given $\varepsilon\left(0<\varepsilon<\frac{1-\rho\left(g_{1}\right)}{2}\right)$, by Lemma 3.2 we obtain

$$
\begin{aligned}
& \left|\frac{g_{1}(z+c)}{g_{1}(z)}\right| \leq \exp \left(r^{\rho\left(g_{1}\right)-1+\varepsilon}\right)=\exp (o(1)), \\
& \left|\frac{g_{1}(z)}{g_{1}(z+c)}\right| \leq \exp \left(r^{\rho\left(g_{1}\right)-1+\varepsilon}\right)=\exp (o(1))
\end{aligned}
$$


outside a finite logarithmic measure $E$. As $z_{k}$ satisfies $\left|g_{1}\left(z_{k}\right)\right|=M\left(r_{k}, g_{1}\right),\left|z_{k}\right|=r_{k} \notin E$, $r_{k} \rightarrow \infty$, we deduce by (3.4) and (3.5) that

$$
\begin{aligned}
\left|A\left(z_{k}\right)\right|= & \left|\frac{P\left(z_{k}\right)}{P\left(z_{k}+c\right)} \frac{g_{1}\left(z_{k}+c\right)}{g_{1}\left(z_{k}\right)}+\frac{P\left(z_{k}+c\right)}{P\left(z_{k}\right)} \frac{g_{1}\left(z_{k}\right)}{g_{1}\left(z_{k}+c\right)}-\frac{B\left(z_{k}\right) P\left(z_{k}\right) P\left(z_{k}+c\right)}{g_{1}\left(z_{k}\right) g_{1}\left(z_{k}+c\right)}-2\right| \\
\leq & \left|\frac{P\left(z_{k}\right)}{P\left(z_{k}+c\right)} \frac{g_{1}\left(z_{k}+c\right)}{g_{1}\left(z_{k}\right)}\right|+\left|\frac{P\left(z_{k}+c\right)}{P\left(z_{k}\right)} \frac{g_{1}\left(z_{k}\right)}{g_{1}\left(z_{k}+c\right)}\right| \\
& +\left|\frac{B\left(z_{k}\right) P\left(z_{k}\right) P\left(z_{k}+c\right)}{M\left(r_{k}, g_{1}\right)^{2}} \frac{g_{1}\left(z_{k}\right)}{g_{1}\left(z_{k}+c\right)}\right|+2 \\
\leq & M,
\end{aligned}
$$

where $M$ is some finite constant, a contradiction, since $\operatorname{deg} A(z)>0$. Hence we have $\rho(g) \geq 1$.

Next, we prove that $\max \left\{\lambda(g), \lambda\left(\frac{1}{g}\right)\right\}=\rho(g)$. If $B(z) \not \equiv 0$, then we set

$$
P(z, g)=(g(z+c)-g(z))^{2}-A(z) g(z+c) g(z)-B(z) .
$$

Since $P(z, 0)=-B(z) \not \equiv 0$, by Lemma 3.1 we deduce that

$$
N\left(r, \frac{1}{g}\right)=T(r, g)+S(r, f)
$$

Hence $\lambda(g)=\rho(g)$.

If $B(z) \equiv 0$, then (2.1) can be reduced to

$$
(g(z+c)-g(z))^{2}=A(z) g(z+c) g(z)
$$

Next, we prove that $\max \left\{\lambda(g), \lambda\left(\frac{1}{g}\right)\right\}=\rho(g)$. Suppose, on the contrary to the assertion, that $\max \left\{\lambda(g), \lambda\left(\frac{1}{g}\right)\right\}=\alpha<\rho(g)$. We next divide the proof into the following two cases.

Case 1. Suppose that $\rho(g)=1$. Then we obtain

$$
g(z)=m(z) \exp ^{q z+p}
$$

where $q \neq 0$ and $p$ are constants, and $m(z)$ is a meromorphic function such that $\rho(m)=$ $\alpha<1$. Substituting (3.6) into (2.1), we obtain

$$
\left(m(z+c) \exp ^{q(z+c)+p}-m(z) \exp ^{q z+p}\right)^{2}=A(z) m(z+c) \exp ^{q(z+c)+p} m(z) \exp ^{q z+p} .
$$

By computing (3.7) we obtain

$$
\begin{gathered}
m^{2}(z+c) \exp ^{2 q c+2 p} \exp ^{2 q z}+m^{2}(z) \exp ^{2 p} \exp ^{2 q z} \\
=(A(z)+2) m(z) m(z+c) \exp ^{q c+2 p} \exp ^{2 q z}
\end{gathered}
$$

that is,

$$
(A(z)+2) \exp ^{q c+2 p} \exp ^{2 q z}=\frac{m(z+c)}{m(z)} \exp ^{2 q c+2 p} \exp ^{2 q z}+\frac{m(z)}{m(z+c)} \exp ^{2 p} \exp ^{2 q z} .
$$


By Lemma 3.2 we obtain

$$
\begin{aligned}
& \left|\frac{m(z+c)}{m(z)}\right| \leq \exp \left(r^{\rho(m)-1+\varepsilon}\right)=\exp (o(1)), \\
& \left|\frac{m(z)}{m(z+c)}\right| \leq \exp \left(r^{\rho(m)-1+\varepsilon}\right)=\exp (o(1))
\end{aligned}
$$

outside a finite logarithmic measure. By (3.9) and (3.10), as $|z| \rightarrow \infty$, we obtain

$$
\begin{aligned}
\left|(A(z)+2) \exp ^{q c+2 p}\right| & =\left|\frac{m(z+c)}{m(z)} \exp ^{2 q c+2 p}+\frac{m(z)}{m(z+c)} \exp ^{2 p}\right| \\
& \leq\left|\frac{m(z+c)}{m(z)} \exp ^{2 q c+2 p}\right|+\left|\frac{m(z)}{m(z+c)} \exp ^{2 p}\right| \leq M_{1}
\end{aligned}
$$

outside a finite logarithmic measure, where $M_{1}$ is a finite constant. This is impossible, since $\operatorname{deg} A(z)>0$.

Case 2. Suppose that $\rho(g)>1$. Then

$$
g(z)=m(z) \exp ^{l(z)}
$$

where $l(z)$ is a polynomial such that $\rho(g)=\operatorname{deg} l(z)>1$, and $m(z)$ is a meromorphic function such that $\rho(m)<\rho(g)$. Substituting (3.11) into (2.1), we obtain

$$
\left(m(z+c) \exp ^{l(z+c)}-m(z) \exp ^{l(z)}\right)^{2}=A(z) m(z+c) \exp ^{l(z+c)} m(z) \exp ^{l(z)} .
$$

Let

$$
l(z)=p_{k} z^{k}+p_{k-1} z^{k-1}+\cdots+p_{1} z+p_{0}
$$

where $p_{k} \neq 0$. Then

$$
\begin{aligned}
& l(z+c)=p_{k} z^{k}+\left(c k p_{k}+p_{k-1}\right) z^{k-1}+Q(z), \\
& l(z+c)-l(z)=\left(c k p_{k}\right) z^{k-1}+Q_{1}(z),
\end{aligned}
$$

where $Q(z)$ and $Q_{1}(z)$ are polynomials of degree at most $k-2$. Equalities (3.12) and (3.14) imply that

$$
m^{2}(z+c) \exp ^{2 c k p_{k} z^{k-1}+2 Q_{1}(z)}+m^{2}(z)=(A(z)+2) m(z+c) m(z) \exp ^{c k p_{k} z^{k-1}+Q_{1}(z)},
$$

that is,

$$
\begin{aligned}
\mid \exp ^{2 c k p_{k} z^{k-1}+2 Q_{1}(z) \mid} & =\left|-\frac{m^{2}(z)}{m^{2}(z+c)}+(A(z)+2) \frac{m(z)}{m(z+c)} \exp ^{c k p_{k} z^{k-1}+Q_{1}(z)}\right| \\
& \leq\left|\frac{m^{2}(z)}{m^{2}(z+c)}\right|+\left|(A(z)+2) \frac{m(z)}{m(z+c)} \exp ^{c k p_{k} z^{k-1}+Q_{1}(z)}\right|
\end{aligned}
$$


By Lemma 3.2 we obtain

$$
\left|\frac{m(z)}{m(z+c)}\right| \leq \exp \left(r^{\rho(m)-1+\varepsilon}\right)
$$

outside a possible set of finite logarithmic measure $E$. As $|z|=r \notin E \cup[0,1]$, and $r \rightarrow \infty$, we deduce by (3.15) and (3.16) that

$$
\begin{aligned}
& \left|\exp ^{2 c k p_{k} z^{k-1}+2 Q_{1}(z)}\right| \\
& \quad \leq \exp \left(2 r^{\rho(m)-1+\varepsilon}\right)+\left|r^{M} \exp \left(r^{\rho(m)-1+\varepsilon}\right) \exp ^{c k p_{k} z^{k-1}+Q_{1}(z)}\right|,
\end{aligned}
$$

where $M$ is a positive constant.

We can find a sequence $\left\{z_{k}\right\}\left(\left|z_{k}\right| \rightarrow \infty\right)$ such that $\left|z_{k}\right|=r_{k} \notin E \cup[0,1]$, and $c p_{k} z_{k}^{k-1}=$ $\left|c p_{k}\right| r_{k}^{k-1}$ as $r_{k} \rightarrow \infty$. We obtain

$$
\left|\exp ^{2 c k p_{k} z_{k}^{k-1}+2 Q_{1}\left(z_{k}\right)}\right|=\exp ^{2 k\left|c p_{k}\right| r_{k}^{k-1}}\left|\exp ^{Q_{1}\left(z_{k}\right)}\right| \geq \exp ^{\frac{3}{2} k\left|c p_{k}\right| r_{k}^{k-1}} .
$$

By (3.17) and (3.18), for any given $\varepsilon\left(0<\varepsilon<\frac{k-\rho(m)}{2}\right)$, we obtain

$$
\exp ^{\frac{3}{2} k\left|c p_{k}\right| r_{k}^{k-1}} \leq \exp \left(2 r_{k}^{\rho(m)-1+\varepsilon}\right)+r_{k}^{M} \exp \left(r_{k}^{\rho(m)-1+\varepsilon}\right) \exp ^{c k p_{k} r_{k}^{k-1}} \leq \exp ^{\frac{6}{5} k\left|c p_{k}\right| r_{k}^{k-1}}
$$

which is impossible. Hence we proved that $\max \left\{\lambda(g), \lambda\left(\frac{1}{g}\right)\right\}=\rho(g)$. Theorem 2.1 is thus proved.

\section{Proof of Theorem 2.2}

Suppose that (2.2) has a rational solution $g(z)$ and has poles $l_{1}, l_{2}, \ldots, l_{k}$. Hence $g(z)$ can be represented as

$$
g(z)=\frac{H(z)}{K(z)}=\sum_{j=1}^{k}\left[\frac{t_{j s_{j}}}{\left(z-l_{j}\right)^{s_{j}}}+\cdots+\frac{t_{j_{1}}}{\left(z-l_{j}\right)}\right]+b_{0}+b_{1} z+\cdots+b_{r} z^{r}
$$

where $b_{0}, \ldots, b_{r}, t_{j_{j}}, \ldots, t_{j 1}$ are constants.

(i) If $d>f$ and $d-f$ is an even number, then (2.2) and (4.1) imply that

$$
\left(\frac{H(z+c)}{K(z+c)}-\frac{H(z)}{K(z)}\right)^{2}-\frac{H(z+c)}{K(z+c)} \frac{H(z)}{K(z)}=\frac{D(z)}{F(z)}
$$

Let $\operatorname{deg} H(z)=h$ and $\operatorname{deg} K(z)=k$. Suppose $h<k$. Then

$$
\lim _{z \rightarrow \infty} \frac{H(z+c)}{K(z+c)}=0, \quad \lim _{z \rightarrow \infty} \frac{H(z)}{K(z)}=0 .
$$

From (4.2) with (4.3) we obtain

$$
\frac{D(z)}{F(z)}=\left(\frac{H(z+c)}{K(z+c)}-\frac{H(z)}{K(z)}\right)^{2}-\frac{H(z+c)}{K(z+c)} \frac{H(z)}{K(z)} \rightarrow 0
$$

This is impossible, since $\frac{D(z)}{F(z)} \rightarrow \infty$ as $z \rightarrow \infty$. 
Suppose $h=k$. Then

$$
\lim _{z \rightarrow \infty} \frac{H(z+c)}{K(z+c)}=\beta, \quad \lim _{z \rightarrow \infty} \frac{H(z)}{K(z)}=\beta,
$$

where $\beta \in \mathbb{C} \backslash\{0\}$. Relations (4.2) and (4.4) yield that

$$
\frac{D(z)}{F(z)}=\left(\frac{H(z+c)}{K(z+c)}-\frac{H(z)}{K(z)}\right)^{2}-\frac{H(z+c)}{K(z+c)} \frac{H(z)}{K(z)} \rightarrow-\beta^{2},
$$

a contradiction, since $\frac{D(z)}{F(z)} \rightarrow \infty$ as $z \rightarrow \infty$. Hence, we obtain that $h>k$. So $b_{r} \neq 0(r \geq 1)$. As $z \rightarrow \infty$, we have

$$
\begin{aligned}
& g(z)=b_{r} z^{s}(1+o(1)), \quad g(z+c)=b_{r} z^{s}(1+o(1)), \\
& \frac{D(z)}{F(z)}=\alpha z^{d-f}(1+o(1)),
\end{aligned}
$$

where $\alpha \in \mathbb{C} \backslash\{0\}$. As $z \rightarrow \infty$, by (4.2) and (4.5) we can deduce

$$
-b_{r}^{2} z^{2 r}(1+o(1))=\alpha z^{d-f}(1+o(1))
$$

Relation (4.6) implies that

$$
h-k=r=\frac{d-f}{2} .
$$

If $d=f$, then, as $z \rightarrow \infty$, we obtain

$$
\frac{D(z)}{F(z)}=\alpha(1+o(1))
$$

where $\alpha \in \mathbb{C} \backslash\{0\}$. If $h<k$, then using the similar method as before, we can obtain a contradiction. If $h>k$, then $b_{r} \neq 0(r \geq 1)$. By (4.2), as $z \rightarrow \infty$, we obtain

$$
-b_{r}^{2} z^{2 r}(1+o(1))=\alpha z^{d-f}(1+o(1))=\alpha(1+o(1)),
$$

a contradiction. Hence $h=k$, that is,

$$
h-k=0=\frac{d-f}{2} .
$$

(ii) We next consider the case $d<f$. Suppose that $h>k$. Then $b_{r} \neq 0(r \geq 1)$. Using the similar method as before, as $z \rightarrow \infty$, by (4.2) we obtain that

$$
3 b_{r}^{2} z^{2 r}(1+o(1))=0
$$

a contradiction.

If $h=k$, then using the similar method as before, we obtain

$$
\frac{D(z)}{F(z)}=\left(\frac{H(z+c)}{K(z+c)}-\frac{H(z)}{K(z)}\right)^{2}-\frac{H(z+c)}{K(z+c)} \frac{H(z)}{K(z)} \rightarrow \beta^{2} \neq 0 \quad \text { as } z \rightarrow \infty
$$


which is a contradiction, since $\frac{D(z)}{F(z)} \rightarrow 0$ as $z \rightarrow \infty$. Hence $h<k$. Substituting $g(z)=\frac{H(z)}{K(z)}$ into (2.2), we have

$$
\begin{aligned}
& F(z) H^{2}(z+c) K^{2}(z)-3 F(z) H(z) H(z+c) K(z) K(z+c)+F(z) H^{2}(z) K^{2}(z+c) \\
& \quad=D(z) K^{2}(z) K^{2}(z+c) .
\end{aligned}
$$

Since

$$
\begin{aligned}
& \operatorname{deg}\left(F(z) H^{2}(z+c) K^{2}(z)-3 F(z) H(z) H(z+c) K(z) K(z+c)+F(z) H^{2}(z) K^{2}(z+c)\right) \\
& \quad=f+2 h+2 k, \\
& \operatorname{deg}\left(D(z) K^{2}(z) K^{2}(z+c)\right)=d+4 k .
\end{aligned}
$$

From this and from (4.8) we have

$$
h-k=\frac{d-f}{2} .
$$

\section{Acknowledgements}

The author would like to thank the referee for his/her helpful suggestions and comments. The work was supported by the NNSF of China (No. 10771121, 11401387), the NSF of Zhejiang Province, China (No. LQ 14A010007), the NSFC Tianyuan Mathematics Youth Fund (No. 11226094), the NSF of Shandong Province, China (No. ZR2012AQ020 and No. ZR2010AM030), and the Fund of Doctoral Program Research of Shaoxing College of Art and Science (20135018).

\section{Competing interests}

The author declares that he has no competing interests.

\section{Author's contributions}

Author read and approved the final manuscript.

\section{Publisher's Note}

Springer Nature remains neutral with regard to jurisdictional claims in published maps and institutional affiliations.

Received: 11 June 2017 Accepted: 10 October 2017 Published online: 17 October 2017

\section{References}

1. Steinmetz, N: Eigenschaften eindeutiger Lösungen gewöhnlicher Differentiagleichungen im Komplexen. Ph.D. thesis, Karlsruhe (1978)

2. Bank, S, Kaufman, R: On the growth of meromorphic solutions of the differential equation $\left(y^{\prime}\right)^{m}=R(z, y)$. Acta Math. $144,223-248(1980)$

3. Ishizaki, K, Korhonen, R: Meromorphic solutions of algebraic difference equations. Submitted

4. Hayman, WK: Meromorphic Functions. Clarendon Press, Oxford (1964)

5. Yang, CC, Yi, HX: Uniqueness of Meromorphic Functions. Kluwer, Dordrecht (2003)

6. Chiang, YM, Feng, SJ: On the growth of logarithmic differences, difference quotients and logarithmic derivatives of meromorphic functions. Trans. Am. Math. Soc. 361, 3767-3791 (2009)

7. Chen, ZX: On growth, zeros and poles of meromorphic solutions of linear and nonlinear difference equations. Sci. China Math. 54, 2123-2133 (2011)

8. Chen, ZX, Huang, ZB, Zhang, RR: On difference equations relating Gamma function. Acta Math. Sci. 31B(4), 1281-1294 (2011)

9. Halburd, RG, Korhonen, R: Difference analogue of the lemma on the logarithmic derivative with applications to difference equations. J. Math. Appl. 314, 477-487 (2006)

10. Ishizaki, K: On difference Riccati equations and second order linear difference equations. Aequ. Math. 81, 185-198 (2011)

11. Laine, I, Yang, CC: Clunie theorems for difference and q-difference polynomials. J. Lond. Math. Soc. 76(2), 556-566 (2007)

12. Liu, Y: Meromorphic solutions of certain difference equations of first order. Aequ. Math. 87(3), 309-323 (2014)

13. Liu, K, Cao, TB: Entire solutions of Fermat type q-difference differential equations. Electron. J. Differ. Equ. 2013, Article ID 59 (2013)

14. Qi, XG, Yang, LZ: Properties of meromorphic solutions of q-difference equations. Electron. J. Differ. Equ. 2015, Article ID 59 (2015)

15. Whittaker, JM: Interpolatory Function Theory. Cambridge Tracts in Mathematics and Mathematical Physics, vol. 33. Stechert-Hafner, New York (1964) 
16. Chiang, YM, Feng, SJ: On the Nevanlinna characteristic of $f(z+\eta)$ and difference equations in the complex plane. Ramanujan J. 16, 105-129 (2008)

17. Halburd, RG, Korhonen, R: Finite order solutions and the discrete Painlevé equations. Proc. Lond. Math. Soc. 94 , 443-474 (2007)

Submit your manuscript to a SpringerOpen ${ }^{\circ}$ journal and benefit from:

- Convenient online submission

- Rigorous peer review

- Open access: articles freely available online

- High visibility within the field

- Retaining the copyright to your article

Submit your next manuscript at $\boldsymbol{~ s p r i n g e r o p e n . c o m ~}$ 